\title{
Diagnostic Imaging
}

National Cancer Institute

\section{Source}

National Cancer Institute. Diagnostic Imaging. NCI Thesaurus. Code C16502.

Any method that uses a visual display of structural or functional patterns of organs or tissues for diagnostic evaluation. 